\title{
Highly Efficient Blue OLEDs Using Ter(9,9-diarylfluorene)s With Ambipolar Carrier Transport Properties
}

\author{
Y. -T. Lin ${ }^{1}$, T.-L. Liu ${ }^{1}$, W. -Y. Hung ${ }^{1}$, P. -Y. Hsieh ${ }^{1}$, C.-C. Wu ${ }^{1 *}$ \\ K.-T. Wong ${ }^{2}$
}

${ }^{1}$ Department of Electrical Engineering, Graduate Institute of Electro-Optical Engineering, and Graduate Institute of Electronics Engineering, National Taiwan University, Taipei,

Taiwan 10617, Republic of China

${ }^{2}$ Department of Chemistry, National Taiwan University, Taipei, Taiwan 10617, Republic of China

*Tel: +886-2-23635251 ext 346, Fax: +886-2-23677467 e-mail: chungwu@cc.ee.ntu.edu.tw

Organic light-emitting devices (OLEDs) have been intensively investigated for their potential applications in high-efficiency, low operating voltage, and full-color large-area flat-panel displays. To achieve full-color displays, blue-emitting materials and devices with high efficiency, good color purity, and good thermal stability are essential and highly desired. Here we report the intriguing carrier transport properties of amorphous ter(9,9-diarylfluorene)s (Fig. (a)), in which unusual nondispersive ambipolar carrier transport properties and high electron mobility of $>10^{-3} \mathrm{~cm}^{2} / \mathrm{V} \cdot \mathrm{s}$ were observed for the first time for amorphous molecular semiconductors. We also report highly efficient and bright blue heterostructure OLEDs based on these of terfluorenes.

The ter(9,9-diarylfluorene)s exhibit under investigation very high glass transition temperatures $(\mathrm{Tg})$ of $>200^{\circ} \mathrm{C}$, good thermal stability, and high thin-film photoluminescent (PL) quntum yields of $\sim 90 \%$ in pure blue. These compounds are able to form homogeneous and stable amorphous films by thermal evaporation, a basic requirement for materials to be used in OLEDs.

The time-of-flight (TOF) transient photocurrent technique was used to characterize the charge transport in films. The terfluorenes show the intriguing carrier transport properties. In these amorphous terfluorene film, unusual nondispersive ambipolar carrier transport properties and high electron mobility of $>10^{-3} \mathrm{~cm}^{2} / \mathrm{V} \cdot \mathrm{s}$ were observed for the first time for amorphous molecular semiconductors (Fig. (b)).

The multilayer OLEDs using these highly efficient blue-emitting terfluorene were fabricated on ITO-coated glass substrates. A 50 -nm-thick film of the terfluorenes served as the hole-transport layer and the emitting layer. A 37-nm-thick electron-transport layer of 1,3,5-tris( $N$-phenylbenzimidazol-2-yl)benzene (TPBI) was used to inject and transport electrons into the EML. The cathode was prepared by sequential deposition of a thin $\mathrm{LiF}$ layer $(0.5 \mathrm{~nm})$ and a thick $\mathrm{Al}(150 \mathrm{~nm})$.

The EL of the devices show pure blue emission from these terfluorenes. The rather low turn-on voltage of $\sim 3 \mathrm{~V}$, defined as the voltage where EL emission becomes detectable, and low operation voltage $\left(100 \mathrm{~cd} / \mathrm{m}^{2}\right.$ at $\sim 6 \mathrm{~V}, 1000 \mathrm{~cd} / \mathrm{m}^{2}$ at $\sim 8 \mathrm{~V}$ ) of these devices suggest these terfluorenes also function well as hole transporters. High EL external quantum efficiency of $2.5-3 \%$ photon/electron and high brightness over $5000 \mathrm{~cd} / \mathrm{m}^{2}$ were observed for these devices (Fig. (c)), consistent with the high thin-film PL quantum yields. High PL/EL quantum efficiencies in conjunction with carrier transport properties render these terfluorenes a very interesting and promising class of optoelectronic materials.

(a)

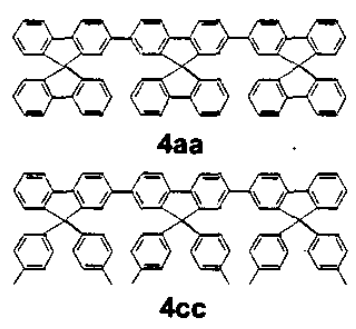

(b)

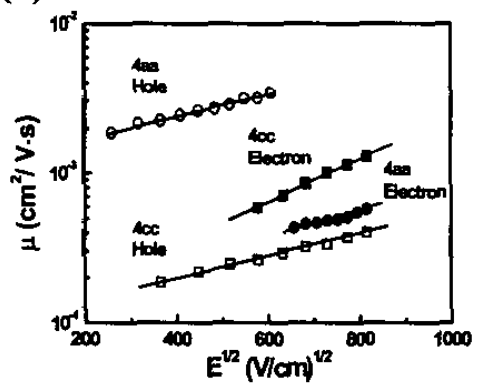

(c)

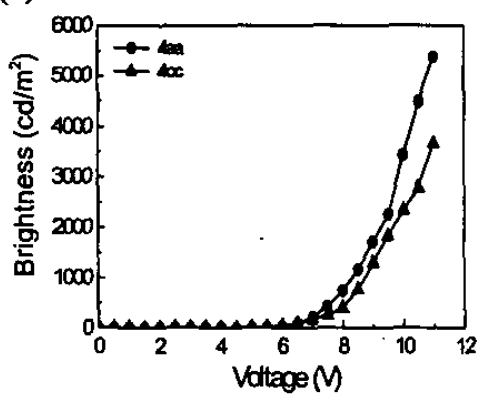

Fig. (a) Chemical structures of ter(9,9-diarylfluorene)s:4aa and 4cc. Fig. (b) Electron and hole mobilities of compounds $4 \mathrm{cc}$ and $4 \mathrm{aa}$ vs. square root of the electric field. Fig. (c) L-V characteristics of the devices with the terfluorene derivatives as emitting material. Device structures: ITO/PEDT:PSS (300 $\AA) /$ terfluorene $4 \mathrm{aa}$ or $4 \mathrm{cc}(500 \AA) / \mathrm{TPBI}(370 \AA) / \mathrm{LiF}(5 \AA) / \mathrm{Al}$. 